\title{
Multislice computer tomography (MSCT) for the optimisation of transcatheter aortic valve implantation (TAVI)
}

Carl J. Schultz and Peter P.T. de Jaegere

Department of Cardiology, Erasmus Medical Centre, Rotterdam,

The Netherlands

Address for correspondence:

Carl Schultz

Department of Cardiology

Erasmus MC

PB 412

3000 CA Rotterdam

The Netherlands

Email:

c.schultz@erasmusmc.nl

\section{INTRODUCTION}

Severe symptomatic aortic stenosis is found in $1 \%$ of patients aged 65 to 75 and 5\% of patients aged over 75 years.(I) Surgical aortic valve replacement (SAVR) is a life saving treatment, but for the approximately one third of patients who are ineligible for SAVR the prognosis is poor with $<50 \%$ survival within I year. ${ }^{(2,3)}$ Transcatheter aortic valve implantation (TAVI) is a new treatment option for patients who are not eligible for surgery due to high levels of co-morbidity. In this patient group the multicentre randomised PARTNER trial demonstrated that TAVI improved prognosis and quality of life when compared to medical therapy. ${ }^{(3)}$

In contrast to open surgery, which proceeds under direct vision, TAVI relies on imaging. Pre-procedural imaging is used to: Select patients who are anatomically suitable for TAVI; determine the vascular access route; size (sizing helps match prosthesis size to patient anatomy); guide the implantation procedure; and to evaluate the result. In the majority of procedures where patients received a TAVI prosthesis thus far contrast angiography (CA) and 2-D echocardiography - either transthoracic (TTE) or transoesophageal (TEE) - were used to execute all these steps. ${ }^{(4,5)}$ However, the aortic root has a complex 3-D anatomy, including crown-shaped leaflet attachments and a non-circular basal plane or annulus, which imposes limitations on 2-D imaging modalities such as CA or TTE/ TEE. In contrast 3-D imaging modalities such as multislice computer tomography (MSCT), cardiac magnetic resonance imaging (CMRI)
ABSTRACT

Transcatheter aortic valve replacement (TAVI) is a novel therapy that is increasingly used to treat patients with severe aortic stenosis at high risk for surgical valve replacement. Patient selection, procedural planning and evaluation all require a detailed analysis of patient anatomy. The role and added value of multislice computer tomography (MSCT) is described as a 3-D imaging modality that enables a comprehensive evaluation of the complex 3-D anatomy of the aortic root, planning of all potential transcatheter access routes and evaluation of prosthesis anatomy interactions post-implantation. SAHeart 20 I2; 9:14-25

and 3-D TEE can evaluate all anatomical aspects of the aortic root. ${ }^{(6-8)}$ MSCT is particularly attractive for the evaluation of anatomy because it provides the user with a 3-D virtual representation that can be viewed from any angle or cut-plane after acquisition. What follows is a review of the role of MSCT pre-and post-TAVI.

\section{DIAGNOSIS OF SEVERE AORTIC STENOSIS}

The diagnosis of severe aortic stenosis is typically based on measurements obtained from transthoracic echocardiography (TTE). The most frequently used measurement is a peak transvalvular flow velocity $>4 \mathrm{~m} / \mathrm{s}$ or alternatively an aortic valve area (AVA) $<1.0 \mathrm{~cm}^{2}$, as calculated from the continuity equation, or an indexed aortic valve area $<0.6 \mathrm{~cm}^{2} / \mathrm{m}^{2}$. In cases where alternative or ancillary measurements are needed AVA is measured by either planimetry on MSCT or CMRI. ${ }^{(910)}$ Many studies have reported a reasonably good correlation between the AVAs from MSCT planimetry and the continuity equation (TTE).(II) Yet, a limitation of the continuity equation is the requirement of a left ventricular outflow tract (LVOT) diameter measurement and the assumption of a circular LVOT for the calculation of LVOT area. This is because studies using 3-D imaging modalities including MSCT have demonstrated that the LVOT is oval shaped, a fact which cannot be appreciated on 2-D echocardiography. ${ }^{(10,12,13,)}$ In 3 separate studies the assumption of circularity of the LVOT was abandoned when using the continuity equation and instead the LVOT area measured on MSCT was used to replace the LVOT diameter from TTE for the calculation of AVA. $(14,15,16)$ These studies reported that the correlation between MSCT planimetered AVA and TTE calculated AVA improved substantially. 
Interestingly the majority of studies that examined the diagnostic accuracy of MSCT planimetry for the diagnosis of severe aortic stenosis used as comparator TTE AVA based on the continuity equation. (II) This can be viewed as a limitation given that the gold standard for the diagnosis of aortic stenosis in patients with preserved left ventricular function is peak transvalvular flow velocity from TTE. There are also technical limitations with measuring AVA by planimetry including obtaining a true short axis viewing plane when using TEE and signal loss caused by calcium. Ultimately it is the physiological limitation in flow that causes symptoms and reduces prognosis in aortic stenosis so that diagnostic parameters incorporating flow have a fundamental advantage over AVA planimetry, which is purely anatomical.

A proof of concept study recently described a technique for measuring transaortic peak flow velocity on MSCT.(17) The diagnostic accuracy of AVA and indexed AVA from MSCT planimetry was found to be moderate when compared to a gold standard of transvalvular peak flow obtained with TTE. MSCT derived peak flow velocity and showed a better sensitivity and specificity (respectively 100\% and 76\%) for the diagnosis of severe aortic stenosis than did aortic valve area (respectively $74 \%$ and $76 \%$ ) or indexed aortic valve area (respectively $74 \%$ and 65\%). Whether this technique may be useful in patients with low flow and a preserved ejection fraction has not yet been studied.

The diagnosis of severe aortic stenosis is often more complex in patients with poor ventricular function. This is because patients with a low ejection volume may not generate a high transvalvular gradient even in the presence of severe aortic stenosis, so called low flow, low gradient aortic stenosis. Alternatively, in patients with non-stenosed aortic leaflets the ejection fraction may be insufficient to fully open the aortic valve, so called pseudostenosis. ${ }^{(18)}$ Dobutamine stress echocardiography is generally used to differentiate true aortic stenosis from pseudostenosis by increasing the transvalvular flow. A recent study reported an association between the degree of aortic root calcification (AVC) and aortic stenosis severity in a validation cohort of 179 patients with preserved left ventricular function (LVF), which was then tested as a diagnostic marker in a testing cohort of 49 patients with impaired LVF. ${ }^{(19)}$ In the testing cohort an AVC threshold of $>165$ I Agatston units, when compared to a gold standard of dobutamine stress echocardiography, had a sensitivity of $95 \%$ and a specificity of $89 \%$ for the detection of severe aortic stenosis. The authors concluded that AVC measured on MSCT may help to identify patients with a low ejection fraction who may benefit from TAVI or SAVR.

\section{ELIGIBILITY}

Pre-procedural imaging is used to evaluate the suitability of the aortic root for the deployment and secure lodgement of one of the TAVI prosthesis types and sizes as well as the feasibility of transcatheter delivery of the prosthesis (Figure I). There are 2 commercially available prostheses namely the Edwards SAPIEN balloon expandable device (ESD) and the Medtronic Corevalve self-expanding device (MCS) (Figure 2, 3). The design and deployment features have been extensively described. ${ }^{(4,5,20)}$ Potential candidates for TAVI are screened based on anatomical eligibility guidelines, which are device-specific due to the differences in the design and geometry between the MCS and ESD (Figure I). The eligibility guidelines provided by industry have recently been summarised. (21)

\section{Aortic root dimensions}

The features of the aortic root that are evaluated during screening are shown in Figure I. An integrated interpretation is helpful, for example: The combination of a low origin of the ostium of the left main stem in combination with a relatively small sinus of Valsalva dimension may risk occlusion of the left main stem, because the deployment of the TAVI prosthesis displaces the calcified native leaflets, in contrast to SAVR where decalcification precedes implantation. On the other hand, provided that the sinus of Valsalva is wide enough to accommodate the displaced calcium, a low origin of the left main stem is unlikely to cause a problem. Due to the hour glass shape of the MCS the narrowest section of the frame will be positioned at the level of the coronary ostia, provided that a satisfactory depth of implantation is obtained following device deployment, a design feature that probably reduces the risk of coronary obstruction. The dimensions of the aortic annulus or basal plane are used to determine whether the aortic root can accommodate one of the TAVI prosthesis sizes.

Sizing

The aortic annulus or basal plane is defined anatomically as the virtual ring with 3 anchor points at the nadirs of the 3 leaflets of the crown shaped aortic valve ${ }^{(2,23)}$ (Figure 2). The dimensions of the aortic annulus are used to select the prosthesis size for which the patient may be eligible. The MCS comes in three sizes based on the nominal inflow diameter. The $26 \mathrm{~mm}$ inflow diameter is meant for an annulus of $20-23 \mathrm{~mm}$ and the $29 \mathrm{~mm}$ for an annulus of $23-27 \mathrm{~mm}$ and the recently introduced $31 \mathrm{~mm}$ inflow for an annulus of $27-29 \mathrm{~mm}$. The Edwards prosthesis comes in sizes 23 and $26 \mathrm{~mm}$ respectively meant for annuli of $18-21 \mathrm{~mm}$ and $22-24 \mathrm{~mm}$ and a $29 \mathrm{~mm}$ device, which can be delivered only via the transapical route, designed for annuli of $25-29 \mathrm{~mm}$. The differences in size between the ESD and the MCS means that the majority of patients will be eligible for one of the two prostheses types. ${ }^{(24)}$

Although the sizing guidelines are straightforward the measurement of the aortic annulus diameter by non-invasive imaging is not as simple as might be implied. Studies using 3-D imaging modalities 


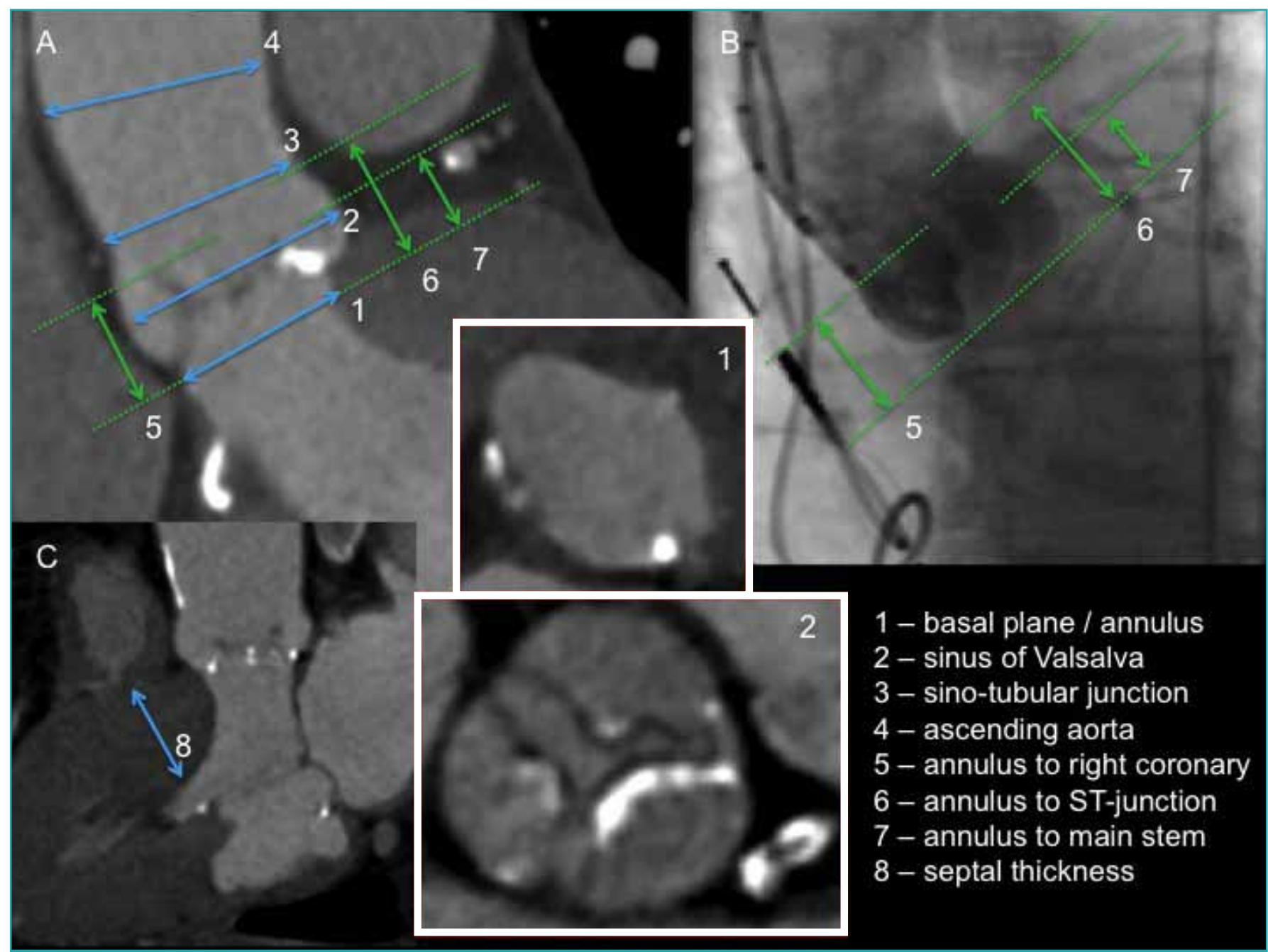

FIGURE I: Anatomical eligibility criteria for TAVI are all evaluable on MSCT

Panel A:A MSCT oblique coronal view shows diameter measurements indicated in blue and height measurements in green. Panel B shows a corresponding view on contrast aortography. Panel $C$ shows the oblique sagittal view. The insets are short-axis images at the levels indicated allowing planimetry of the aortic valve area (level 2) and detailed evaluation of the aortic annulus or basal plane (level I).

including MSCT, CMRI and 3-D TTE/TEE have shown that the aortic annulus is not round but oval in shape $\mathrm{e}^{(10,12,13,25)}$ (Figure 3). In one study using MSCT to evaluate the aortic root in 75 patients who were candidates for TAVI an oval annulus was seen in approximately $78 \%$ of patients. ${ }^{(26)}$ By implication a minimum and maximum diameter could be measured and the difference between the 2 was on average $6.5 \mathrm{~mm}$ (95\% confidence interval 5.7-7.2). The TTE parasternal long axis view and TEE 3-chamber, both of which are routinely used for sizing, allow only measurement of an oblique sagittal diameter, which approximates the minimum diameter of the aortic annulus.(27) The coronal, which approximates the maximum diameter, cannot be measured on TTE/TEE ${ }^{(6,28)}$ (Figure 3). The mean annulus diameters derived from any of the arithmetic mean, perimeter or area barely differ from each other and fall in between the minimum and maximum. ${ }^{(26)}$ The annulus diameter measured a TTE PLAX view or TEE 3 chamber view falls in between the mean and the minimum diameter, and the coronal diameter from contrast aortography falls in between the mean and maximum diameters. ${ }^{(27,28)}$ The differences between the imaging modalities represent anatomically different dimensions of a complex structure rather than discrepancies, (Figure 3), whereas a lesser contribution may be expected from measurement variability and -method. Figure 3 shows how these measurements relate to one another based on an axial image of the aortic annulus. The use of the different diameter measurements has substantial implications for the selection of prosthesis size. One study examined sizing for the ESD by either MSCT or TTE and TEE in 45 patients. (27) If sizing were based on MSCT mean diameter $38 \%$ of patients would not have been eligible for an ESD (due to the annulus being too large) whereas respectively $24 \%$ and $33 \%$ were ineligible based on TTE and TEE. The authors concluded, based on the results of their current practice, that sizing should be based on TTE/TEE. 
Another study examining the hypothetical effects of sizing for the MCS by different MSCT diameter measurements in 75 patients reported that $26 \%$ to $39 \%$ of patients would not be eligible for a MCS if sizing were based on the minimum and maximum diameters ${ }^{(26)}$ respectively. Sizing based on any of the mean annulus diameter measurements resulted in $90 \%$ of patients being eligible for the MCS. The lower rate of eligibility due to an overly large annulus based on MSCT mean annulus diameter in the study of the ESD when compared to the study with the MCS is explained by the fact that at the time of the study, only the 2 smaller sizes of ESD were available. Another study compared different diameter measurements of the aortic annulus on pre-TAVI MSCT (minimum, maximum, coronal, sagittal, mean from cross-sectional area [CSA]) with the inflow diameter achieved post-implantation with the ESD in 24 patients. (29) The post-implantation ESD diameter correlated strongly with mean diameter from native annulus CSA $(r=0.84, p<0.01)$ whereas the correlation with all the other diameter measurements were moderate to poor (all $r<0.65$ ). Another study that obtained MSCT pre- and post-TAVI with the MCS in 30 patients also reported that the post-implantation cross-sectional area achieved at the inflow of the MCS frame was most similar to the mean diameter and the CSA of the native annulus preimplantation. ${ }^{(30)}$ Despite these interesting observations there are no randomised data on which approach to sizing gives the best outcome. An important point is that the recent availability of more prosthesis sizes increases the proportion of eligible patients but also the potential adverse effects of a sizing error. Data that suggest an effect of sizing on outcome, but not which sizing strategy to use, is discussed in a later section.



FIGURE 2: Sizing for TAVI is based on the dimensions of the aortic annulus

Sizing for both Edwards SAPIEN (A) and Medtronic CoreValve (D) devices is based on the "diameter" of the aortic annulus. Anatomically the aortic valve is crown-shaped (B) and the aortic annulus is not an anatomical entity but rather a virtual ring with 3 anchor points at the nadirs of the 3 aortic leaflets (green line, C). On angiography the level of the aortic annulus can be localised before (E) and after (F) TAVI. The inset (G) shows on a short-axis view from MSCT that the aortic annulus is usually oval-shaped so that more than one diameter measurement is required to describe its dimensions. 


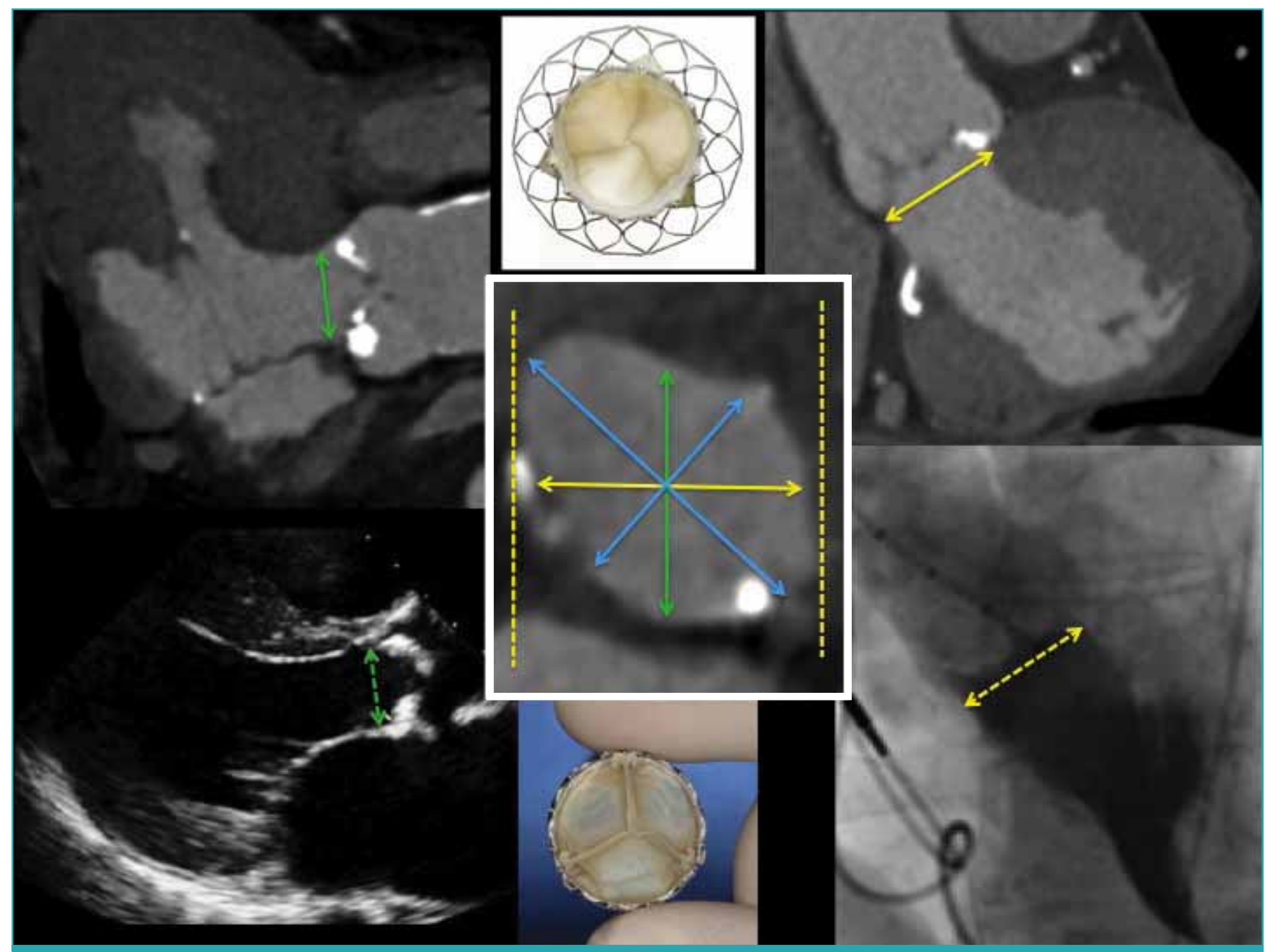

FIGURE 3: MSCT facilitates understanding of which dimensions of the aortic annulus may be measured with different imaging modalities The left column shows an oblique sagittal view on MSCT (top) corresponding to the parasternal long axis view on transthoracic- and the 3 chamber view on transoesophageal echocardiography (bottom). The right column shows an oblique coronal view on MSCT (top) corresponding to an anteroposterior type view on contrast angiography (bottom). On a short-axis view of the aortic annulus (inset, middle column) the diameter measurements obtained from echocardiography (green) and angiography (yellow) are indicated as well as the true minimum and maximum dimensions (blue), which are not readily appreciable on echocardiography or angiography in approximately one third of patients.

\section{Vascular access}

All 3 available prosthesis sizes of the MCS 3rd generation and the $23 \mathrm{~mm}$ ESD are deliverable through an I8F transvascular sheath, whereas the $26 \mathrm{~mm}$ ESD requires a $19 \mathrm{~F}$ sheath. Transapical delivery is possible for all the ESD-XT prosthesis sizes and is the only route of access possible with the $29 \mathrm{~mm}$ ESD. The gold standard for the assessment of vascular access route is invasive angiography, which may require multiple-contrast injections to obtain a vascular overview of the aortic root, arch abdominal aorta and iliac-femoral arteries. ${ }^{(31)}$

Multiple studies have shown that MSCT is an excellent diagnostic tool for the detection of significant stenosis in the peripheral vasculature. $^{(32)}$ MSCT is increasingly being used for the more demanding role of screening vascular access routes for TAVI. ${ }^{(33,34)}$ The advantage is that an overview of all potential vascular access routes, including transfemoral, -subclavian or -apical may be obtained, ${ }^{(35)}$ (Figure 4). In a combined scanning protocol the vascular overview can be obtained immediately following a scan of the heart without requiring additional contrast.

With modern scanning techniques, such as prospective ECG synchronised high pitch (flash), only an incremental increase in radiation dose is required above that of the heart scan. ${ }^{(35)}$ In addition to information on vascular luminal diameter MSCT provides a detailed overview of the 3 dimensional tortuosity and calcification of the vascular tree. All 3 these factors have to be considered when choosing the access route in order to deduce permissibility for the 


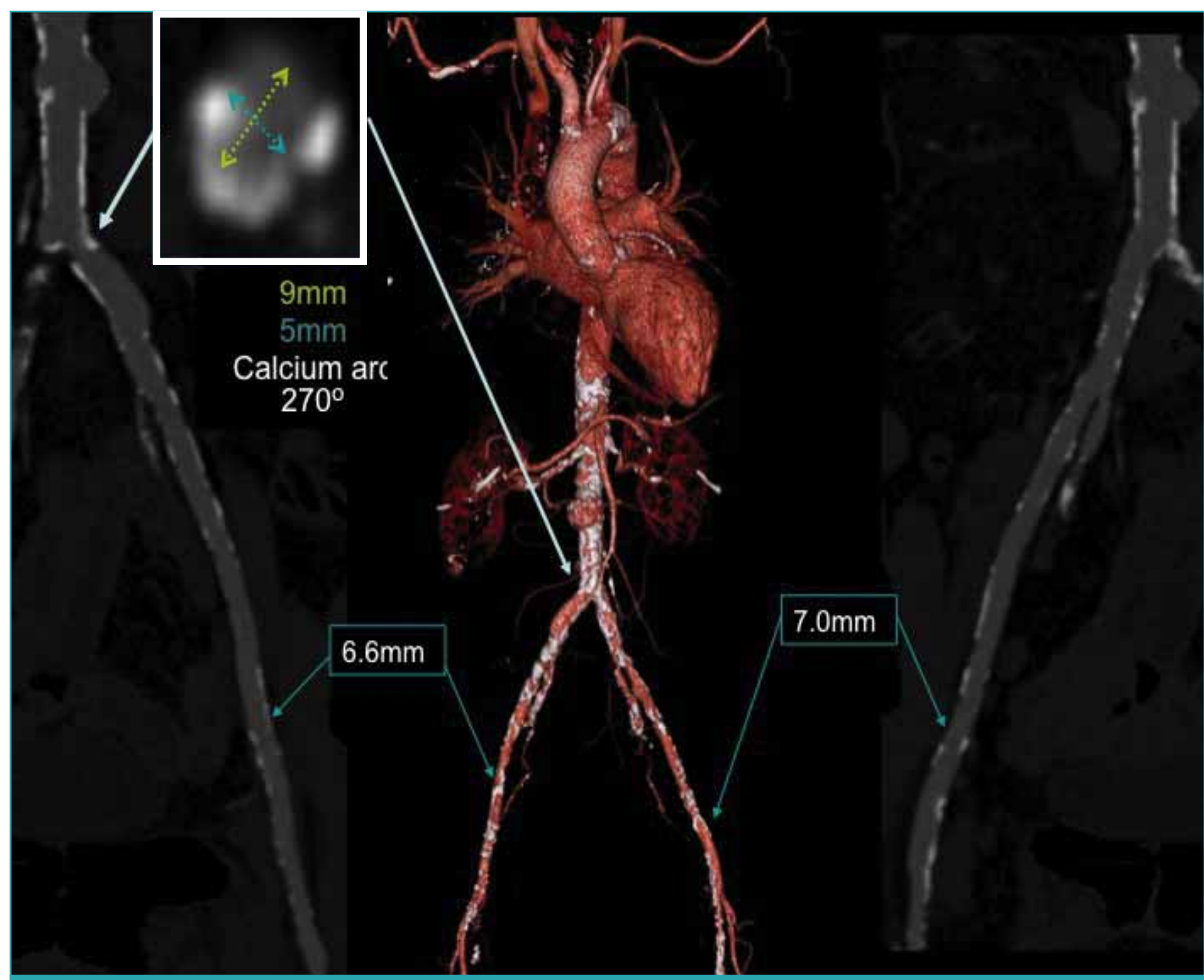

FIGURE 4: A vascular overview obtained from MSCT

A 3-dimensional reconstruction shows an overview of tortuosity and calcification (middle). A detailed interrogation of lumen diameter and the amount and degree of circumferentiality of calcification is performed on multiplanar reformatted images (left, right and inset showing a short-axis view).

relatively rigid undeployed TAVI prostheses, ${ }^{(34,31)}$ (Figure 4, 5). In addition to planning access via the transfemoral and subclavian arteries MSCT allows evaluation of transapical and direct access routes, including the optimal ribspace and direction of puncture for true percutaneous procedures, (Figure 6). TTE is at present much more widely used for determining the point of entry for transapical access. On the other hand, TTE may not be useful for direct aortic access due to the interposition of lung tissue although experience with this approach is limited.

An important point is that MSCT tends to exaggerate the thickness of calcification. This means that in the presence of dense calcification, in particular if it is semi-circumferential or worse, the lumen diameter may be either over- or underestimated depending on the measurement technique. Caution is warranted with near circumferential calcium even with a clearly permissible lumen diameter due to the increased rigidity of the vessel and the consequent resistance to passage of a relatively large device. A study of 129 patients who received either an ESD ( 102 patients) or a MCS (27 patients) investigated the determinants of major vascular complications as defined by Vascular Academic Research Consortium (VARC) criteria. (34) Major determinants in multivariate analysis were the ratio of sheath outer diameter to femoral artery minimum lumen diameter (SFAR) and femoro-iliac artery calcification. A SFAR of >1.05 was also associated with increased mortality. 


\section{PROCEDURAL GUIDANCE}

Contrast angiography (CA) is likely to remain the standard imaging technique for guidance for the foreseeable future. Two aspects that may improve procedural guidance include the use of the optimal projection (OP) and new developments in software aimed at facilitating positioning during device deployment.

\section{Optimal projection}

The OP is defined as the C-arm angulation that will show the 3 aortic sinuses aligned on one plane and separated so that all 3 sinuses are clearly distinguishable. The optimal projection can be obtained from pre-procedural MSCT. ${ }^{(36,37,38)}$ In brief this is done by first setting up a short-axis plane of the aortic annulus, then parallel shifting it to the level of the coaptation of the aortic leaflets. Following this, the cross-hairs are rotated so that one plane runs along the coaptation line of the non- and left-coronary and through the middle of the right coronary sinus and finally by reading off the viewing angle of the orthogonal (oblique coronal) plane. If pre-procedural MSCT is not available multiple contrast injections may be needed with adjustment of the $\mathrm{C}$-arm angulation to obtain the correct view. The use of the OP during TAVI avoids overlap and consequent confusion of the sinuses. In one small study the use of the OP obtained from pre-procedure MSCT resulted in a better final implantation depth of the ESD when compared to 20 subsequent patients who did not have pre-procedure MSCT. ${ }^{(36)}$ The optimal projection also makes possible accurate evaluation of depth of implantation, which is important in evaluating the potential causes and subsequent corrective steps required (balloon dilatation if the depth is optimal, a valve-in-valve procedure if it is positioned too deep) in the event that significant paravalvular aortic regurgitation (PAR) is seen post-deployment. ${ }^{(39)}$

\section{HOW TO INTERROGATE AN MSCT PRE-TAVI}

A step-by-step method for defining the aortic annulus on MSCT and the different measurements has been described using a


FIGURE 5: Evaluation of tortuosity

A 3-D vascular overview showing significant tortuosity of the femora-iliac vessels and the thoraco-abdominal aorta (left). The multiplanar reformatted images allow measurement of lumen diameter of the subclavian artery (top, middle). As the artery crosses the first rib there is a severe angulation that can be appreciated in the poster-superior view (green), but not in the antero-posterior view (red). 



FIGURE 6: Planning of transapical and other unusual approaches on MSCT

Planning for a transapical approach shows the optimal rib space and direction of puncture (top row). In patients with no other options direct aortic access via a mini-thoracotomy has been described and the potential catheter path can be planned with MSCT (bottom row).

conventional MSCT workstation as well as dedicated TAVI-planning software that reduces the amount of user input required and speeds up the analysis process. ${ }^{(6)}$

\section{EVALUATION}

After device release the depth of implantation, frame expansion and degree of aortic regurgitation are of interest. MSCT allows a detailed evaluation of frame expansion, asymmetry and apposition to surrounding tissue on short axis images (Figure 7). This applies to both the ESD and the MCS. ${ }^{(30,40)}$ Although MSCT of the frame is not routinely required for clinical management it may be valuable in understanding the cause of complications in selected cases for example where there is a high residual gradient, unexplained aortic regurgitation or a question about coronary impingement. ${ }^{(41,42,43)}$

\section{THE EVOLVING ROLE OF DIFFERENT IMAGING \\ MODALITIES}

A more detailed understanding is emerging of anatomical features, in addition to those described in the industry defined eligibility guidelines, which may affect the outcome of TAVI. These data are from case series and have focused on predictors of intermediate outcomes including paravalvular aortic regurgitation, the need for balloon post-dilatation, device dislodgement and new conduction abnormalities or pacing requirement.

Paravalvular aortic regurgitation and the need for balloon post-dilatation

Haemodynamically significant aortic regurgitation after TAVI is poorly tolerated and is immediately corrected during the implantation procedure by either post-dilatation or, if the cause is a 

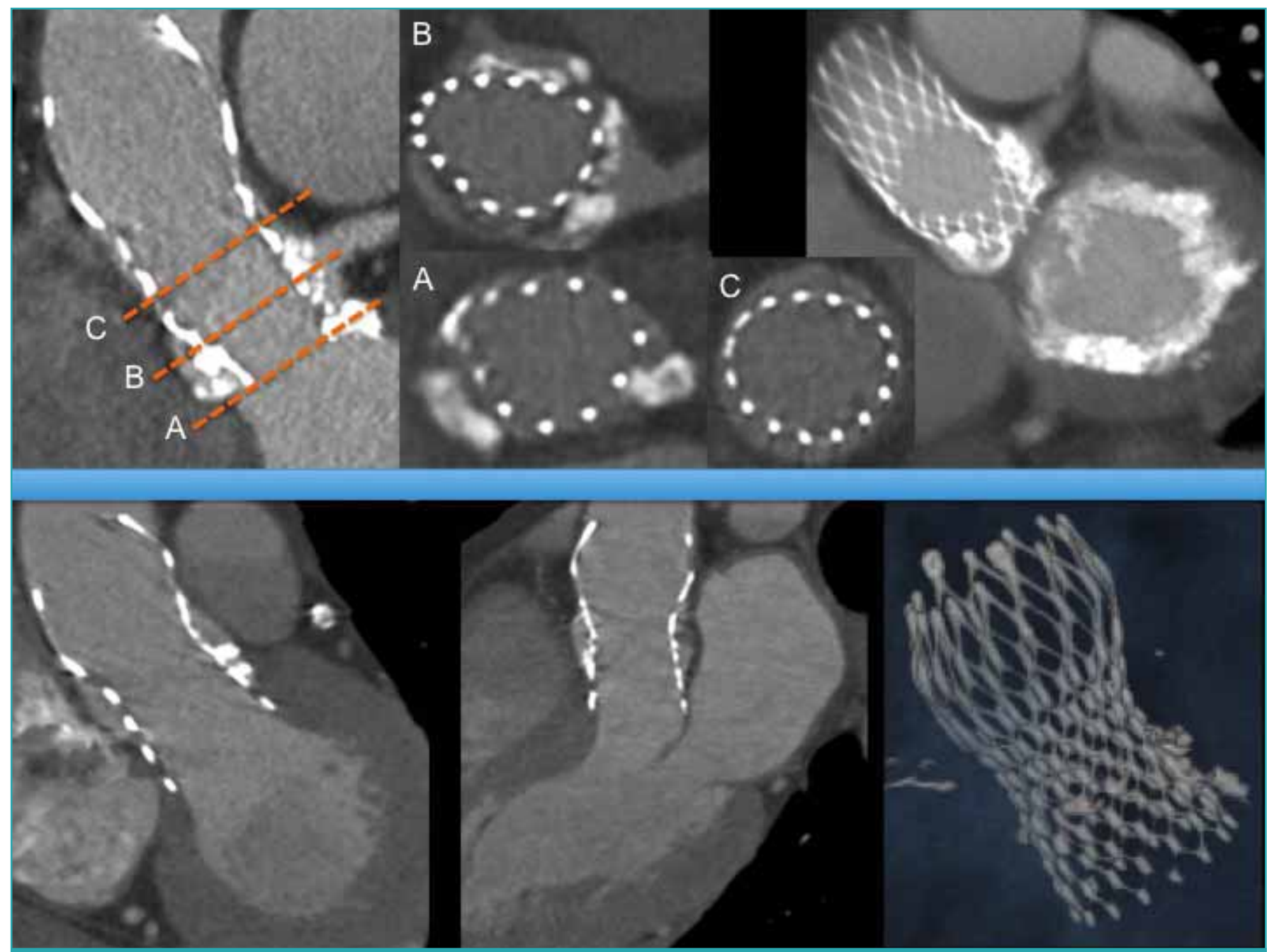

\section{FIGURE 7: MSCT after TAVI}

The top row shows a Medtronic CoreValve device implanted in a patient with very severe calcification of the aorta-mitral fibrous continuity. The coronal view (left) shows a relatively high implant. Short-axis views at different levels as indicated show calcification causing frame asymmetry and malapposition at the inflow (I), a chunk of calcium coming close to, but not obstructing the left main stem (2), and a near normal circularity of the frame and expansion at the functionally important level of coaptation of the leaflets (3). The interaction between the frame and calcification of the aortic valve and mitral annulus are shown in a maximum intensity projection (right).

The bottom row shows an early implant of a Medtronic CoreValve device that was relatively deep as seen in the coronal view (left) and restricts mitral valve opening as seen in the sagittal view (middle).A 3-D reconstruction of the frame from MSCT is shown on the right.

too deep position of the implanted prosthesis, implantation of a second prosthesis (valve-in-valve). Residual paravalvular aortic regurgitation of a mild or more severe degree is common after TAVI occurring in between 15 and $50 \%$ of cases. ${ }^{(44,45,46,47.48)}$ Although mild to moderate PAR after SAVR is not thought to adversely affect haemodynamics a recent retrospective multicentre registry found an association of mild to moderate PAR (defined as $22 / 4$ ) with increased mortality in TAVI patients. ${ }^{(46)}$ The data suggested that residual PAR is a marker of an adverse risk profile and it is not clear whether reducing the degree of PAR may also reduce risk of adverse events in the present TAVI population.
The intention of industry guidelines for prosthesis size selection is for some degree of over-sizing to ensure a reasonable force of apposition of the sealing skirt to the surrounding tissue in order to ameliorate PAR and ensure positional stability. Imaging studies of the aortic root using either TTE or MSCT have found that residual PAR was associated with larger aortic annulus dimensions and a lower ratio of nominal prosthesis to annulus diameter, indicating that a lesser degree of over-sizing may be associated with PAR. ${ }^{(44,45,49,50)}$ In these studies there was substantial overlap in the prosthesis to annulus ratio between patients with and without mild to moderate PAR, indicating that other factors also play a role. 
A small study of 54 patients localised the jets of PAR around the MCS prosthesis inflow in relation to the native anatomy and found that PAR was most common on the inside curve of the aortic arch, which is also the left side of the largest diameter of the aortic annulus, and at the aorta-mitral fibrous continuity. ${ }^{(45)}$ In the same study MSCT post-TAVI demonstrated that mal-apposition of the device frame to the surrounding tissue was also more frequent at these two anatomical locations. The authors concluded that the angulation of the aorta in addition to sizing and calcification may contribute to the etiology of PAR after TAVI with an MCS. ${ }^{(45)}$ In other studies mild to moderate PAR was also associated with a higher degree of calcification of the aortic root. ${ }^{(39,50)}$ In patients with a more than moderate degree of PAR immediately after TAVI, balloon post-dilatation is usually the first step to reduce it.

A case series of 100 patients who received a MCS and where postdilatation was performed in 30\% quantified the degree of aortic root calcification on pre-procedural MSCT. In that study an aortic root Agatston score $>3000$ associated with post-dilatation and was positively correlated with the final degree of regurgitation. ${ }^{(39)}$ Another study of 110 patients who received the MCS and where post-dilatation was performed in II cases found that the need for balloon post-dilatation was associated with larger annulus diameter, a lower ratio of prosthesis to annulus size and the degree of calcification of the aortic root or leaflets. ${ }^{(51)}$ In that study the ability to discriminate the need for balloon post-dilatation was poor for the prosthesis to annulus ratio (area under a ROC curve 0.3), moderate for annulus dimensions (area under ROC curve 0.67) and excellent for calcification (area under ROC curve >0.8). ${ }^{(51)}$ These data indicate that calcification is the most-important determinant of significant PAR and the need for post-dilatation and may, in future, lead to the testing of new procedural strategies intended to reduce PAR in patients known to have very dense aortic root calcification.

\section{Device dislodgement}

Late (post-implantation procedure) dislodgement of the TAVI prosthesis is rare. In one case report it was thought to be related to low levels of calcification of the aortic leaflets, poor left ventricular function and possibly to undersizing. ${ }^{(52)}$ Predictors of intraprocedural device dislodgement, which required device retrieval and repeated implantation in the correct position, were investigated in one study of 98 patients. ${ }^{(53)}$ Device dislodgement occurred in 18 of the patients and was associated with larger aortic valve area and a lower degree of aortic root calcification. In multivariate analysis an aortic root calcium threshold of <2 359 (Agatston score) was the only independent predictor for valve dislodgement
(OR 3.I, 95\% confidence interval I.I - 8.8). The authors proposed that, in patients with low levels of aortic root calcification device deployment during rapid pacing may be considered to avoid dislodgement during the deployment stage. ${ }^{(53)}$

New conduction abnormalities or pacing requirement New conduction abnormalities requiring implantation of a permanent pacemaker are higher after TAVI than after SAVR. The rate of implantation of permanent pacemakers in patients with a MCS ranges from 9 to 39\%. ${ }^{(54-56)}$ Anatomical factors identified on MSCT that were associated with pacemaker requirement include a higher prosthesis to annulus ratio and a higher pre-dilatation balloon to annulus ratio. ${ }^{(55,57)}$ Whether aortic root calcification is associated with pacemaker requirement is controversial.(58,59)

\section{latrogenic membranous VSD}

latrogenic membranous VSD is uncommon after TAVI but may have adverse consequences. ${ }^{(41,60)}$ A case report described the occurrence of iatrogenic VSD following post-dilatation of the TAVI prosthesis with a relatively oversized balloon in a patient with an unusually long ventricular membranous septum. The unusual anatomic finding of a long ventricular extent of the membranous septum was readily discernable on pre-implantation MSCT in a small series of patients. ${ }^{(41)}$

\section{CONCLUSIONS:}

\section{THE EVOLVING ROLE OF IMAGING}

The use of MSCT for planning TAVI has improved our understanding of the role and limitations of commonly used 2-D imaging modalities including CA and TTE/TEE and is contributing to a more detailed understanding of anatomical factors, identifiable on preprocedural imaging that may impact on procedural outcome. Most of these observations come from case series and as such the data need to be confirmed. However, a detailed understanding of patient anatomy obtained from MSCT will increasingly lead to a tailored patient-specific approach to optimise the outcome of TAVI.

\section{Conflict of interest: none declared.}




\section{REFERENCES}

I. Nkomo VT, Gardin JM, Skelton TN, et al. Burden of valvular heart diseases: A population-based study. Lancet. 2006;368: I005-II.

2. lung B. A prospective survey of patients with valvular heart disease in Europe: The Euro Heart Survey on Valvular Heart Disease. European Heart Journal. 2003;24:1231-1243

3. Leon MB, Smith CR, Mack M, et al. Transcatheter aortic-valve implantation for aortic stenosis in patients who cannot undergo surgery. The New England Journal of Medicine. 2010;363:1597-607.

4. Cribier A, Eltchaninoff $\mathrm{H}$, Tron $\mathrm{C}$, et al. Treatment of calcific aortic stenosis with the percutaneous heart valve: Mid-term follow-up from the initial feasibility studies: the French experience. Journal of the American College of Cardiology. 2006;47:1214-23

5. Grube E, Laborde JC, Zickmann B, et al. First report on a human percutaneous transluminal implantation of a self-expanding valve prosthesis for interventional treatment of aortic valve stenosis. Catheterisation and Cardiovascular Intervention. 2005:66:465-9.

6. Schultz CJ, Moelker AD, Tzikas A, et al. Cardiac CT: Necessary for precise sizing for transcatheter aortic implantation. Eurolntervention; 20 I 0;6 Suppl:G6-GI3.

7. Altiok E, Koos R, Schroder J, et al. Comparison of two dimensional and three dimensional imaging techniques for measurement of aortic annulus diameters before transcatheter aortic valve implantation. Heart. 201 I: 1-7.

8. Paelinck BP, Van Herck PL, Rodrigus I, et al. Comparison of magnetic resonance imaging of aortic valve stenosis and aortic root to multimodality imaging for selection of transcatheter aortic valve implantation candidates. The American Journal of Cardiology. 201 1; 108:92-8.

9. Bouvier E, Logeart D, Sablayrolles J-L, et al. Diagnosis of aortic valvular stenosis by multislice cardiac computed tomography. European Heart Journal. 2006; 27:3033-8.

10. Burgstahler C, Kunze M, Löfler C, et al. Assessment of left ventricular outflow tract geometry in non-stenotic and stenotic aortic valves by cardiovascular magnetic resonance. Journal of Cardiovascular Magnetic Resonance. 2006;8:825-9.

II. Abdulla J, Sivertsen J, Kofoed KF, et al. Evaluation of aortic valve stenosis by cardiac multislice computed tomography compared with echocardiography: A systematic review and meta-analysis. The Journal of Heart Valve Disease. 2009; 18:634-43.

12. Doddamani S, Bello R, Friedman M, et al. Demonstration of left ventricular outflow tract eccentricity by real time 3-D echocardiography: Implications for the determination of aortic valve area. Echocardiography (Mount Kisco, N.Y.) 2007:24:860-6

13. Doddamani S, Grushko MJ, Makaryus AN, et al. Demonstration of left ventricular outflow tract eccentricity by 64-slice multidetector CT. The International journal of Cardiovascular Imaging. 2009;25: 175-81.

14. Halpern EJ, Mallya R, Sewell M, et al. Differences in aortic valve area measured with CT planimetry and echocardiography (continuity equation) are related to divergent estimates of left ventricular outflow tract area. AJR. American Journal of Roentgenology. 2009; 192:1668-73.

15. O'Brien B, Schoenhagen P, Kapadia SR, et al. Integration of three dimensional imaging data in the assessment of aortic stenosis: Impact on classification of disease severity. Circulation. Cardiovascular Imaging. 201 I.

16. Utsunomiya $\mathrm{H}$, Yamamoto $\mathrm{H}$, Horiguchi J, et al. Underestimation of aortic valve area in calcified aortic valve disease: Effects of left ventricular outflow tract ellipticity. International Journal of Cardiology. 201 I.

17. Schultz CJ, Papadopoulous SL, Moelker A, et al. Transaortic flow velocity from dual-source MSCT for the diagnosis of aortic stenosis severity. Catheterisation and Cardiovascular Interventions: 201 1:78:127-35.
18. Dumesnil JG, Pibarot P, Carabello B. Paradoxical low flow and/or low gradient severe aortic stenosis despite preserved left ventricular ejection fraction: Implications for diagnosis and treatment. European Heart Journal. 20 10;31:281-9.

19. Cueff C, Serfaty J-M, Cimadevilla C, et al. Measurement of aortic valve calcification using multislice computed tomography: Correlation with haemodynamic severity of aortic stenosis and clinical implication for patients with low ejection fraction Heart. 20I0; 97(9):721-6.

20. Vahanian A, Alfieri O, Al-Attar N, et al. Transcatheter valve implantation for patients with aortic stenosis: A position statement from the European Association of Cardio-Thoracic Surgery (EACTS) and the European Society of Cardiology (ESC), in collaboration with the European Association of Percutaneous Cardiovascular Interventions (EAPCI). European Heart Journal. 2008;29: I 463-70.

21. Delgado $\mathrm{V}$, Ewe $\mathrm{SH}, \mathrm{Ng} A C T$, et al. Multimodality imaging in transcatheter aortic valve implantation: Key steps to assess procedural feasibility. Eurolntervention. 2010;6:643-52.

22. Piazza N, De Jaegere P, Schultz C, et al. Anatomy of the aortic valvar complex and its implications for transcatheter implantation of the aortic valve. Circulation Cardiovascular Interventions. 2008; 1:74-81.

23. Anderson RH. Clinical anatomy of the aortic root. Heart (British Cardiac Society) 2000;84:670-3

24. Jilaihawi $H$, Bonan $R$, Asgar $A$, et al. Anatomic suitability for present and next generation transcatheter aortic valve prostheses: Evidence for a complementary multidevice approach to treatment. JACC. Cardiovascular Interventions. 2010; 3:859-66.

25. Tops LF, Krishnàn SC, Schuijf JD, et al. Non-coronary applications of cardiac multidetector row computed tomography. JACC. Cardiovascular Imaging. 2008; 1:94-106.

26. Schultz CJ, Moelker A, Piazza N, et al. Three dimensional evaluation of the aortic annulus using multislice computer tomography: Are manufacturer's guidelines for sizing for percutaneous aortic valve replacement helpful? European Heart Journal. 2010;31:849-56.

27. Messika-Zeitoun D, Serfaty J-M, Brochet E, et al. Multimodal assessment of the aortic annulus diameter: Implications for transcatheter aortic valve implantation Journal of the American College of Cardiology. 20 10;55: 186-94.

28. Tzikas A, Schultz C], Piazza N, et al. Assessment of the aortic annulus by multislice computed tomography, contrast aortography and trans-thoracic echocardiography in patients referred for transcatheter aortic valve implantation. Catheterisation and Cardiovascular Interventions. 201 1;77:868-75.

29. Blanke P, Siepe M, Reinöhl J, et al. Assessment of aortic annulus dimensions for Edwards SAPIEN Transapical Heart Valve implantation by computed tomography: Calculating average diameter using a virtual ring method. European Journal of Cardio-thoracic Surgery. 2010;38:750-8.

30. Schultz CJ, Weustink A, Piazza N, et al. Geometry and degree of apposition of the CoreValve revalving system with multislice computed tomography after implantation in patients with aortic stenosis. Journal of the American College of Cardiology. 2009;54:91।-8.

31. Eltchaninoff H, Kerkeni M, Zajarias A, et al. Aorta-iliac angiography as a screening tool in selecting patients for transfemoral aortic valve implantation with the Edwards SAPIEN bioprostheses. Eurolntervention. 2009:5:438-42.

32. Met R, Bipat S, Legemate DA, Reekers JA, Koelemay MJW. Diagnostic performance of computed tomography angiography in peripheral arterial disease: A systematic review and meta-analysis. JAMA. 2009;301:415-24

33. Kurra $V$, Schoenhagen $P$, Roselli EE, et al. Prevalence of significant peripheral artery disease in patients evaluated for percutaneous aortic valve insertion: Preprocedural assessment with multidetector computed tomography. The Journal of Thoracic and Cardiovascular Surgery. 2009; 1 37: 1258-64. 
34. Hayashida K, Lefèvre $T$, Chevalier B, et al. Transfemoral aortic valve implantation. JACC: Cardiovascular Interventions. 201 1;4:851-858.

35. Wuest W, Anders K, Schuhbaeck A, et al. Dual source multidetector CTangiography before transcatheter aortic valve implantation (TAVI) using a highpitch spiral acquisition mode. European Radiology. 20 I2;22(1):5 I-8.

36. Gurvitch R, Wood DA, Leipsic J, et al. Multislice computed tomography for prediction of optimal angiographic deployment projections during transcatheter aortic valve implantation. JACC. Cardiovascularlinterventions. 20 I 0;3:1 I 57-65.

37. Kurra V, Kapadia SR, Tuzcu EM, et al. Pre-procedural imaging of aortic root orientation and dimensions: Comparison between $X$-ray angiographic planar imaging and 3-dimensional multidetector row computed tomography. JACC. Cardiovascular Interventions. 2010;3:105-13.

38. Tzikas A, Schultz C, Van Mieghem NM, et al. Optimal projection estimation for transcatheter aortic valve implantation based on contrast-aortography: Validation of a prototype software. Catheterisation and Cardiovascular Interventions. 2010;76:602-7.

39. John D, Buellesfeld L, Yuecel S, et al. Correlation of device landing zone calcification and acute procedural success in patients undergoing transcatheter aortic valve implantations with the self-expanding CoreValve prosthesis. JACC. Cardiovascular Interventions. 2010;3:233-43.

40. Caudron J, Fares J, Hauville C, et al. Evaluation of multislice computed tomography early after transcatheter aortic valve implantation with the Edwards SAPIEN bioprosthesis. The American Journal of Cardiology. 2011 .

4I. Tzikas A, Schultz C, Piazza N, et al. Perforation of the membranous interventricular septum after transcatheter aortic valve implantation. Circulation. Cardiovascular interventions. 2009;2:582-3.

42. Schultz C, Piazza N, Weustink A, et al. How should I treat acute valve regurgitation? Eurolntervention. 2011;7:151-9.

43. Kapadia SR, Svensson L, Tuzcu EM. Successful percutaneous management of left main trunk occlusion during percutaneous aortic valve replacement. Catheterisation and Cardiovascular Interventions: 2009;73:966-72.

44. Détaint D, Lepage $L$, Himbert D, et al. Determinants of significant paravalvular regurgitation after transcatheter aortic valve: implantation impact of device and annulus discongruence. JACC. Cardiovascular Interventions. 2009;2:821-7.

45. Schultz CJ, Tzikas A, Moelker A, et al. Correlates on MSCT of paravalvular aortic regurgitation after transcatheter aortic valve implantation using the Medtronic CoreValve prosthesis. Catheterisation and Cardiovascular Interventions. 201 I.

46. Abdel-Wahab M, Zahn R, Horack M, et al. Aortic regurgitation after transcatheter aortic valve implantation: Incidence and early outcome. Results from the German transcatheter aortic valve interventions registry. Heart. 201 1;97:899-906.

47. Grube E, Schuler G, Buellesfeld L, et al. Percutaneous aortic valve replacement for severe aortic stenosis in high-risk patients using the second- and current thirdgeneration self-expanding CoreValve prosthesis: Device success and 30-day clinical outcome. Journal of the American College of Cardiology. 2007;50:69-76.

48. Clavel M-A, Webb JG, Pibarot P, et al. Comparison of the haemodynamic performance of percutaneous and surgical bioprostheses for the treatment of severe aortic stenosis. Journal of the American College of Cardiology. 2009;53:| 883-9|.

49. Takagi K, Latib A, Al-Lamee R, et al. Predictors of moderate-to-severe paravalvular aortic regurgitation immediately after core valve implantation and the impact of post-dilatation. Catheterisation and Cardiovascular Interventions. 2011 .

50. Koos R, Mahnken AH, Dohmen G, et al. Association of aortic valve calcification severity with the degree of aortic regurgitation after transcatheter aortic valve implantation. International Journal of Cardiology. 2010.
5I. Schultz C, Rossi A, Van Mieghem NM, et al. Aortic annulus dimensions and leaflet calcification from contrast MSCT predict the need for balloon post-dilatation after TAVI with the Medtronic CoreValve prosthesis. Eurolntervention. 20I I;(e-pub ahead of print)

52. Clavel M-A, Dumont $E$, Pibarot $P$, et al. Severe valvular regurgitation and late prosthesis embolisation after percutaneous aortic valve implantation. The Annals of Thoracic Surgery. 2009;87:618-21.

53. Van Mieghem NM, Schultz C, van der Boon R, et al. Incidence, timing and predictors of valve dislodgement during TAVI with the Medtronic CoreValve system. Catheterisation and Cardiovascular Interventions. $201 \mathrm{l}$.

54. Zahn R, Gerckens U, Grube E, et al. Transcatheter aortic valve implantation: First results from a multicentre real world registry. European Heart Journal. 2010.

55. Bleiziffer S, Ruge H, Hörer J, et al. Predictors for new-onset complete heart block after transcatheter aortic valve implantation. JACC. Cardiovascular Interventions. 2010;3:524-30.

56. Piazza N, Onuma $Y$, Jesserun E, et al. Early and persistent intra-ventricular conduction abnormalities and requirements for pacemaking after percutaneous replacement of the aortic valve. JACC. Cardiovascular Interventions. 2008; 1:310-6.

57. Nuis R-J, Van Mieghem NM, Schultz CJ, et al. Timing and potential mechanisms of new conduction abnormalities during the implantation of the Medtronic CoreValve System in patients with aortic stenosis. European Heartjjournal. 2011;32:2067-74.

58. Koos R, Mahnken AH, Aktug $\mathrm{O}$, et al. Electrocardiographic and imaging predictors for permanent pacemaker requirement after transcatheter aortic valve implantation. The Journal of Heart Valve Disease. 201 1;20:83-90.

59. Latsios G, Gerckens U, Buellesfeld L, et al. "Device landing zone" calcification, assessed by MSCT, as a predictive factor for pacemaker implantation after TAVI. Catheterisation and Cardiovascular Interventions. 2010;76:431-9.

60. Al-Attar N, Ghodbane W, Himbert D, et al. Unexpected complications of transapical aortic valve implantation. The Annals of Thoracic Surgery. 2009; 88:90-4. 